\title{
Creencias de directivos escolares: implicancias en el liderazgo pedagógico
}

\author{
María Elena Mellado Hernández \\ Universidad Católica de Temuco - Temuco - Chile \\ Juan Carlos Chaucono Catrinao \\ Universidad Católica de Temuco - Temuco - Chile \\ Carolina Pilar Villagra Bravo \\ Universidad Católica de Temuco - Temuco - Chile
}

\section{Resumen}

El objetivo del artículo consiste en develar las creencias que poseen líderes escolares sobre diseño de enseñanza, proceso de aprendizaje y evaluación, según enfoque pedagógico por transmisión o construcción. El trabajo se suscribe a la investigación educativa. Participan 10 directivos que lideran centros educativos de educación primaria y secundaria, región de La Araucanía, Chile. Para recoger información se aplica un cuestionario de escala tipo Likert y se realiza un grupo de discusión entre los participantes. Los resultados cuantitativos reflejan que coexisten creencias híbridas en el pensamiento directivo desde enfoques pedagógicos por transmisión y construcción. Sin embargo, al analizar el contenido discursivo, emergen las creencias por transmisión. En conclusión, prevalecen creencias directivas con un enfoque pedagógico por transmisión que subyace a la toma de decisiones de estos líderes. Surge la necesidad de una formación directiva centrada en un liderazgo pedagógico que tensione y reconstruya las creencias de directores escolares.

Palabras-clave: Creencias pedagógicas; proceso pedagógico; liderazgo pedagógico.

\section{School leaders' beliefs: implications in the educational leadership}

\begin{abstract}
The aim of this article is to reveal the beliefs that leaders have on the instructional design, learning and assessment process, according to the pedagogical approach by transmission or construction. The work subscribes to the educational research. There, participated 10 principals who lead schools in primary and secondary education, in Araucania region, Chile. To collect the information, a questionnaire Likert scale is applied and is done in a group discussion among participants. The quantitative results show that hybrid beliefs coexist in the principal thinking from pedagogical approaches $\mathrm{b}$ transmission and construction. However, when analyzing the discursive content, transmission beliefs emerge. In conclusion, prevailing the principal beliefs with a pedagogical approach underlying transmission decisions of these leaders. Arises the need of management training focused on pedagogical leadership that stresses and rebuild the beliefs of school principals.
\end{abstract}

Keywords: pedagogical beliefs; educational process; pedagogical leadership.

\section{Crenças de diretores escolares: implicações na liderança pedagógica}

\section{Resumo}

O objetivo deste artigo consiste em revelar as crenças que possuem os líderes escolares sobre projeto de ensino, processo de aprendizagem e avaliação, segundo o enfoque pedagógico por transmissão ou construção. O trabalho se subscreve à investigação educativa. Participam 10 diretores que lideram centros educativos de educação primária e secundária da região da Araucanía, Chile. Para recolher as informações, se aplica um questionário de escala tipo Likert e se realiza um grupo de discussão entre os participantes. Os resultados quantitativos refletem que coexistem crenças híbridas no pensamento diretivo desde os enfoques pedagógicos por transmissão e construção. No entanto, ao analisar o conteúdo discursivo, emergem as crenças por transmissão. Em conclusão, prevalecem as crenças diretivas com enfoque pedagógico por transmissão que subjaz à tomada de decisão destes líderes. Surge a necessidade de uma formação diretiva centrada na liderança pedagógica que tencione e reconstrua as crenças de diretores escolares.

Palavras-chave: Crenças pedagógicas; processo pedagógico; liderança pedagógica. 


\section{Introducción}

Durante la última década, el liderazgo pedagógico se ha convertido en una competencia esencial para mejorar la calidad educativa. En este sentido, Leithwood, Harris, \& Hopkins (2008) y Barber \& Mourshed (2007) sitúan al liderazgo directivo como el segundo factor interno de la escuela que más afecta el aprendizaje de niños y niñas. Por consiguiente, surge la necesidad de un mayor conocimiento pedagógico por parte del equipo directivo para liderar los centros escolares con foco en el desarrollo profesional docente y el aprendizaje del estudiantado.

En este contexto, distintos estudios han constatado que prevalece en los centros escolares la mirada de gestión escolar desde una perspectiva tradicional centrada en la administración con una visión disociada entre lo pedagógico y organizacional (Garbanzo \& Orozco, 2010; Rivera, 2010; Krichesky \& Murillo, 2011). Asimismo, Anderson (2010) plantea que el líder educativo sigue evadiendo la responsabilidad del aprendizaje del estudiante, delegando esta tarea en otros miembros del equipo directivo, en el profesorado, en los estudiantes y sus familias. En otras palabras, en la actualidad aún encontramos en los centros escolares equipos directivos que adolecen del conocimiento pedagógico necesario para asumir un efectivo liderazgo para el aprendizaje.

En Chile, ha surgido en la última década un conjunto de políticas educativas en favor del liderazgo escolar, que se han traducido en diversos instrumentos que orientan la gestión de prácticas directivas efectivas. En este sentido, el Ministerio de Educación (2015) ha elaborado un nuevo Marco para la Buena Dirección y el Liderazgo Escolar que considera el desarrollo de la profesionalización y el fortalecimiento del rol directivo. Este instrumento otorga relevancia al liderazgo pedagógico, emplaza al directivo a desarrollar capacidades profesionales docentes al interior del centro educativo. Además, de la exigencia de asegurar calidad en la implementación curricular y propiciar condiciones para el mejoramiento de la enseñanza y el aprendizaje. Esta mirada pedagógica, que la política demanda del directivo, requiere de colaboración y aprendizaje mutuo entre docentes y directivos para conformar, en conjunto, una comunidad profesional de diálogos pedagógicos reflexivos que puedan develar y tensionar las creencias del proceso de enseñanza aprendizaje (Macbeath \& Nempster, 2009; Muñoz \& Farfán, 2009; Anderson, 2010; Elmore, 2010; Bolívar, 2010). En otras palabras, el líder escolar debería comprender los principios pedagógicos que subyacen al proceso de aula para orientar la mejora de sus prácticas directivas y del desempeño profesional docente.

Diversas investigaciones han sostenido la necesidad de explorar en las representaciones implícitas que sostienen directivos escolares con el propósito de ahondar sobre las creencias que manifiestan, en las cuales confluyen constructos que usan cuando lideran y orientan el accionar pedagógico del profesorado. Estas creencias se construyen por diferentes referentes epistemológicos, valorativos y afectivos, mimetizados por un sincretismo de conocimientos y saberes profesionales (Clark, 2008; Martínez, Montero, \& Pedrosa,
2009; Díaz, Martínez, Roa, \& Sanhueza, 2010; Contreras, 2010; Rojas, 2014). Desde esta lógica, Mellado \& Chaucono (2016) afirman que en el pensamiento docente directivo aparentemente coexisten distintos enfoques. Sin embargo, al profundizar en el estudio y comprensión de las creencias, generalmente prevalecen representaciones tradicionales que dan sentido y guían las prácticas directivas.

En tal sentido, conocer cómo conciben el proceso pedagógico los equipos directivos podría explicar las creencias subyacentes y cómo estas se desarrollan en la forma de liderar los centros escolares. En este marco, los avances investigativos han develado la posibilidad de asociar las creencias que poseen directivos a los modos en los cuales basan su práctica dando sentido a una serie de acciones que planifican para orientar el trabajo pedagógico del profesorado en el aula. Siguiendo este marco, Díaz y cols. (2010) manifiestan la necesidad de esclarecer las creencias con el propósito de comprender la concepción que poseen sobre los conocimientos pedagógicos. En otras palabras, las creencias subyacen al discurso y pueden evidenciarse a través de enfoques por transmisión o construcción.

En los últimos años, diversas investigaciones (Kaplan, 2004; Prieto, 2008; Gómez, Muñoz, Silva, González, Guerra, \& Valenzuela, 2014; Mellado \& Chaucono, 2015) han manifestado que el enfoque pedagógico por transmisión considera el diseño de enseñanza como un proceso de instrucción vertical que se construye solamente desde el currículum prescrito, obviando principios del diseño universal para el aprendizaje que tienen como propósito maximizar la igualdad de oportunidades e inclusión de todo el estudiantado. Asimismo, este enfoque por transmisión tributa actividades de enseñanza aprendizaje de mera reproducción del conocimiento escolar, que finalmente termina siendo una réplica de la instrucción plasmada en la planificación, sin atender y dar respuesta a la diversidad e interacción que surge espontáneamente en el aula. Además, otorga excesiva relevancia a aspectos estructurales de la clase, al texto escolar y protagonismo del docente. Del mismo modo, distintos autores (Ahumada, 2005; Sanmartí, 2007; Pimienta, 2008), han sostenido que la evaluación desde una perspectiva tradicional tiende a ser relacionada solo con la calificación y asociada al control con sentido meramente punitivo de parte del profesorado. En otras palabras, el enfoque por transmisión considera la evaluación como una instancia para calificar el resultado final del proceso formativo obviando la interacción, los otros agentes y el proceso de retroalimentación.

Por otro lado, diferentes investigadores (Coll, Martín, Mauri, Miras, Onrubia, Solé, \& Zabala, 2007; Martínez y cols., 2009; García, 2010), manifiestan que desde el enfoque por construcción, el diseño y planificación de la enseñanza exige instancias reflexivas sobre el currículum escolar, contexto y características del estudiante para la toma de decisiones pedagógicas pertinentes con el propósito de generar oportunidades significativas de aprendizaje. Desde este mismo enfoque, el proceso de interacción pedagógica en el aula, considera de manera crucial la mediación del docente, quien proporciona espacios y tiempos para el desarrollo integral 
del alumnado. En este sentido, se otorga al estudiante un rol protagónico en la construcción del conocimiento, a partir de sus necesidades, intereses, saberes y experiencias previas. Asimismo, incorpora en su clase las características y particularidades del contexto del alumnado para diversificar las actividades de aprendizaje. En relación a la evaluación, Sanmartí (2007) \& Pimienta (2008) la asumen como una oportunidad de aprendizaje, que cumple un rol formativo que otorga participación a los agentes involucrados en el proceso. En el enfoque por construcción y de acuerdo a los propósitos de la evaluación y la diversidad en el aula, se utilizan diversas estrategias e instrumentos evaluativos que favorezcan una retroalimentación oportuna y pertinente, tanto al estudiante como docente, para la mejora continua del proceso pedagógico.

Dado los antecedentes, queda de manifiesto la necesidad de investigar sobre las creencias pedagógicas de líderes escolares, en atención al rol predominante que tienen en el desarrollo profesional docente, y por ende, en el aprendizaje del estudiante. En tal sentido, el objetivo de este artículo consiste en develar las creencias que poseen directivos escolares sobre el proceso de diseño, enseñanza-aprendizaje y evaluación, según enfoque pedagógico por transmisión o construcción.

\section{Método}

La investigación se suscribe al enfoque de investigación educativa, enmarcándose en un estudio de caso, utiliza una metodología mixta de carácter descriptivo que tiene el objetivo de comprender y aportar conocimiento para la mejora educativa (Bisquerra, 2004; Amaya, 2007).

\section{Participantes}

Los participantes de la investigación fueron seleccionados de forma intencionada. Corresponden a 10 directivos pertenecientes a centros educativos subvencionados por el Estado, que atienden educación primaria y secundaria de la Región de La Araucanía, Chile. Los establecimientos educacionales se ubican en zonas urbanas, cuentan con altos índices de vulnerabilidad y tienen una matrícula promedio de 750 estudiantes.

Las edades del equipo directivo participante fluctúan entre 38 y 65 años de edad, el $60 \%$ corresponde a hombres y el $40 \%$ a mujeres. Estos directivos no poseen formación en liderazgo pedagógico y acumulan un promedio de 14 años de experiencia en cargos directivos. El $80 \%$ tiene título de profesor/a de estado en enseñanza secundaria y un $20 \%$ en enseñanza primaria.

\section{Técnicas e Instrumentos}

Esta investigación ha utilizado la técnica de cuestionario y grupo de discusión para la recolección de informa- ción. En primer lugar, se aplica un cuestionario tipo escala Likert, construido y validado por los investigadores, cuyo objetivo fue develar las creencias del proceso pedagógico de los equipos directivos sobre tres dimensiones referidas al diseño y planificación, enseñanza aprendizaje y evaluación. El instrumento posee validez de contenido por juicio de expertos y se analizó su fiabilidad total elementos por cada dimensión, a través del coeficiente alpha de Cronbach, obteniendo un .81 que indica seguridad y precisión en la medición del constructo. Después del proceso de validez, el instrumento queda conformado por 60 enunciados, de los cuales 18 corresponden a la dimensión de diseño y planificación, 22a enseñanza aprendizaje y 20 pertenecen al proceso evaluativo. Estos enunciados han sido redactados en pares de ítems, asociados tanto a un enfoque pedagógico por transmisión como por construcción, presentándose aleatoriamente en el instrumento.

En segundo lugar, se realiza un grupo de discusión con todas las personas participantes de la investigación, durante 120 minutos. Para propiciar el diálogo se utilizaron preguntas orientadoras de la conversación, similares a los enunciados del cuestionario. La recogida de información se hizo con registros de audio, luego se transcriben los datos para un análisis de contenido con foco en las dimensiones de diseño y planificación, enseñanza aprendizaje y evaluación. Después de 2 meses se repite nuevamente el grupo de discusión para profundizar en algunos aspectos de las dimensiones con el propósito de ampliar la información recogida.

\section{Procedimientos}

En primer lugar, se aplicó el cuestionario al grupo participante de manera colectiva, durante 30 minutos. Luego se revisaron las respuestas y asignaron las puntuaciones obtenidas en el cuestionario, según enfoque pedagógico y respectivas dimensiones del instrumento. Posteriormente se ordenó la información en una planilla Excel para proceder a realizar el análisis de estadística descriptiva que permitieran obtener resultados cuantitativos sobre creencias directivas en relación al enfoque por transmisión y construcción para las dimensiones de diseño y planificación, enseñanza aprendizaje y proceso evaluativo. Finalmente, se contrastaron los resultados de ambos enfoques.

En segundo lugar, se procedió al análisis cualitativo de la información recogida en ambos grupos de discusión. Se comienzó con la transcripción de los registros de audio, luego se realizó un análisis de contenido de la información recurrente en virtud de las categorías preestablecidas del proceso pedagógico, tanto para el enfoque por transmisión como por construcción. Finalmente, se organizó la información encontrada en una representación gráfica. De esa forma se esquematiza el discurso directivo para lograr una mejor comprensión de los hallazgos cualitativos. Para ello, se utilizó el software Cmap Tools que aporta a la manipulación, almacenamiento, recuperación y el abordaje multimedial de los datos. Esta herramienta digital permitió construir mapas 
conceptuales con enlaces para expandir la explicación de los contenidos o buscar información relacionada.

\section{Resultados}

En este apartado se presentan los resultados tendientes a develar las creencias sobre diseño de enseñanza, proceso de aprendizaje y evaluación que asumen directivos escolares desde un enfoque por transmisión y construcción. Se comienza exponiendo los resultados cuantitativos y luego se presentan los hallazgos cualitativos del estudio.

\section{Resultados cuantitativos de las creencias sobre el proceso pedagógico de directivos escolares}

A continuación, la figura1 presenta las creencias sobre el diseño de enseñanza, proceso de aprendizaje y evaluación que ha manifestado el cuerpo directivo, según enfoque pedagógico por transmisión y construcción.

Los resultados de la figura 1, muestran que el equipo directivo participante del estudio manifiesta creencias sobre el proceso pedagógico que resultan híbridas, desde una interpretación asociada a los enfoques por transmisión y construcción. Es decir, aparecen creencias sobre diseño de enseñanza, proceso de aprendizaje y evaluación que obtienen promedios similares.

Sin embargo, al analizar detalladamente las medias obtenidas en la dimensión de diseño de enseñanza se observa una leve inclinación de las creencias directivas hacia el enfoque pedagógico por construcción. En cambio, la dimensión de evaluación se asocia a creencias desde un enfoque por transmisión.

\section{Hallazgos cualitativos sobre creencias del proceso pedagógico expresadas por directivos escolares}

Los hallazgos que ha develado el análisis de contenido sobre el proceso pedagógico que declaran equipos directivos en relación a dimensiones de diseño y planificación de enseñanza, proceso de aprendizaje y evaluación se organizan en representaciones gráficas.

\section{Dimensión del diseño y planificación de la enseñanza}

Esta dimensión de diseño y planificación de la enseñanza se asume por el equipo directivo de este estudio, como un proceso vertical y descontextualizado del estudiante, con actividades diseñadas principalmente desde los contenidos disciplinares que tienden a reproducir el conocimiento escolardes de el currículum prescrito. A continuación, se presenta la figura 2, que evidencia gráficamente este hecho en particular.

La figura 2 demuestra que este equipo directivo escolar concibe el diseño y planificación de la enseñanza desde un enfoque pedagógico por transmisión. Esta interpretación se justifica desde la comprensión tradicional que tienen los participantes del diseño de la enseñanza explicitada en sus discursos pedagógicos. Estos líderes educativos recurrentemente apelan a la estructura de la clase, actividades reproductivas y el currículum nacional para explicar el proceso de diseño de la enseñanza y planificación de clase. En este sentido, el equipo directivo otorga relevancia excesiva a las estructuras y formatos de planificación, dando énfasis a cuestiones triviales más que a principios pedagógicos del diseño. Además, comentan actividades homogéneas

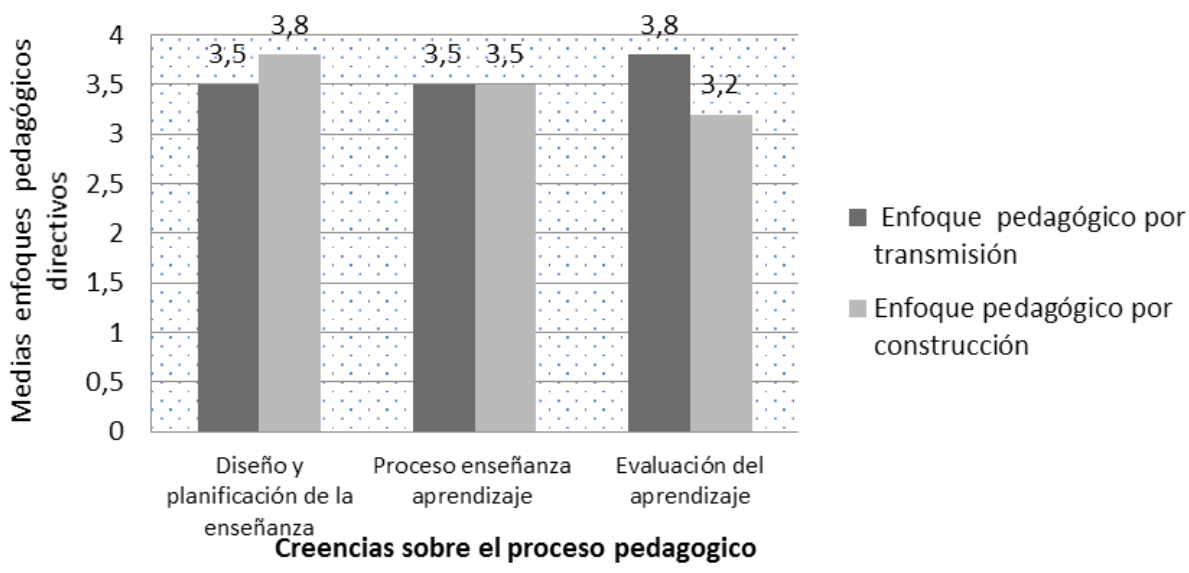

Figura 1. Creencias de directivos escolares sobre proceso pedagógico, según enfoque por transmisión y construcción.

Fuente: Elaboración propia de los autores (2016). 


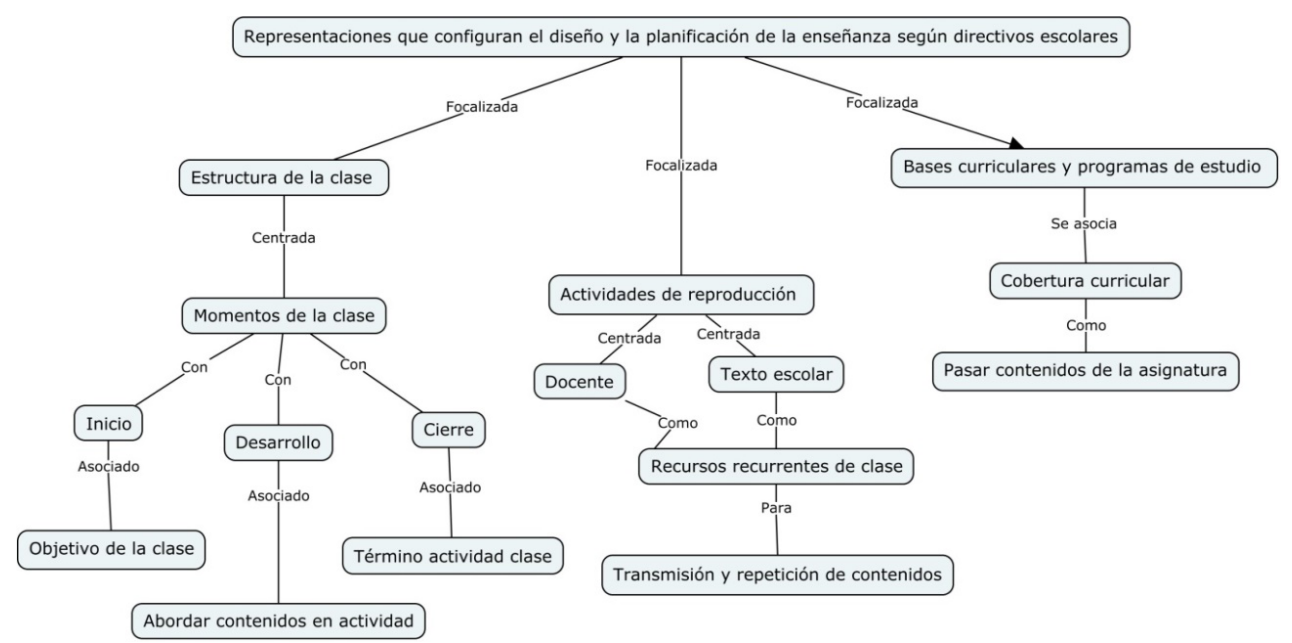

Figura 2. Representaciones sobre el diseño y planificación de la enseñanza según directivos escolares.

Fuente: Elaboración propia de los autores (2016).

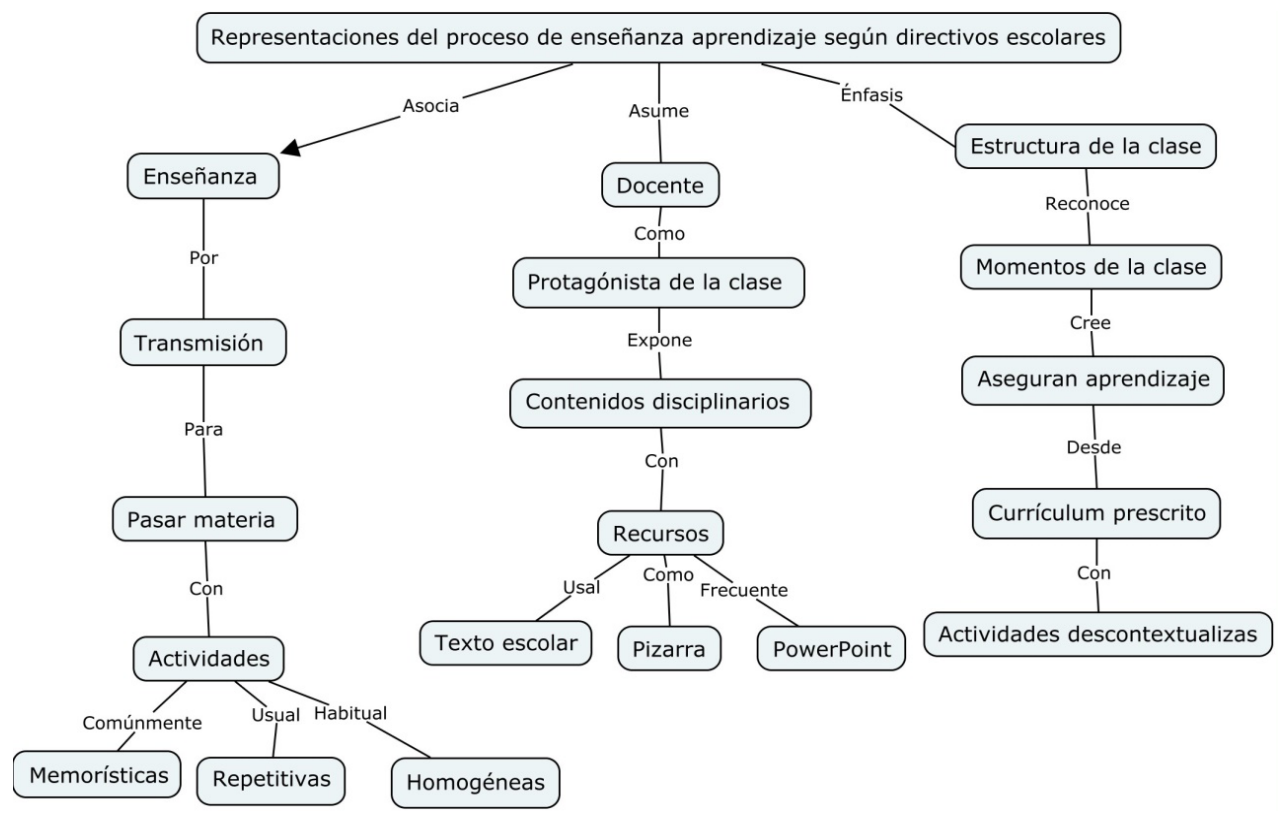

Figura 3. Representaciones sobre el proceso de enseñanza, según directivos escolares.

Fuente: Elaboración propia de los autores (2016).

centradas en el profesorado y texto escolar en desmedro de la consideración del contexto sociocultural del estudiante. Del mismo modo, entienden el diseño solo desde las Bases Curriculares y programas de estudio, asocian comúnmente la cobertura curricular como cubrir la cantidad de contenidos prescritos de la asignatura, así la transmisión se valora positivamente, en la medida que piensan que ahorran tiempo con actividades centradas en la enseñanza y reproducción del conocimiento escolar.

\section{Dimensión del proceso de enseñanza aprendizaje}

Los participantes asumen el proceso de aprendizaje como una práctica centrada en la enseñanza de contenidos disciplinarios del currículum por parte del profesorado. Esto denota una mirada jerárquica del aula y descontextualizada del estudiante. A continuación la figura 3 muestra una gráfica de las representaciones del pensamiento directivo en la dimensión de proceso de enseñanza aprendizaje. 
La figura 3, ejemplifica que este equipo directivo asume el proceso de enseñanza aprendizaje desde un enfoque pedagógico por transmisión. Esto se explica a través de argumentos que develan creencias arraigadas como enseñar desde la transmisión de contenidos por parte del docente, quien utilizaría como recurso principal el texto escolar, la pizarra y presentaciones en PowerPoint. Las actividades que comentan se pueden clasificar en memorísticas, homogéneas y repetitivas. Además, aparece la creencia que una clase con una ordenada estructura asegura el aprendizaje del estudiante.

\section{Dimensión de la evaluación del aprendizaje}

Estos líderes escolares asumen la evaluación como medición de contenidos por parte solo del docente con el propósito de calificar las asignaturas. Se argumenta que esta repetición de contenido en las pruebas es el resultado final del aprendizaje. A continuación, la Figura 4representa gráficamente las creencias del equipo directivo en esta dimensión.

La figura 4, muestra que los participantes respecto de la evaluación comparten el enfoque pedagógico por transmisión. Se observa que asumen la evaluación como resultados de la medición de contenidos al final del proceso de enseñanza. Específicamente, dan énfasis a la evaluación sumativa porque otorga calificaciones necesarias para obtener un promedio de notas por cada asignatura. Estas personas justifican que la prueba es el mejor instrumento para evaluar, consideran que tiene mayores niveles de obje- tividad. La retroalimentación se asume como la revisión de las respuestas de la prueba, responsabilizando al estudiante del error que surge en la evaluación.

\section{Discusión}

Este estudio en particular, da cuenta que directivos escolares al enfrentarse a un conjunto de referentes teóricos, reconocen simultáneamente planteamientos educativos desde enfoques pedagógicos tanto por transmisión como por construcción social del aprendizaje. Esta aparente coexistencia de afirmaciones y juicios que emiten estos equipos directivos sobre el proceso pedagógico, puede entenderse desde diferentes autores, quienes explican que algunas creencias por su naturaleza y origen, estarían más sujetas a controversia que otras. Se trata de un constructo donde confluyen distintos tipos de ideas, asociadas a expectativas y percepciones de un fenómeno, marcadas por la prescripción o el deber ser (Jiménez \& García, 2006; Wanlin, 2009; Gómez, 2010).

En este estudio en particular, las creencias del equipo directivo sobre el diseño de enseñanza, se manifiestan en discursos acotados y restringidos desde el punto de vista teórico y empírico, donde prima un enfoque tradicional que orienta la toma de decisiones al momento de gestionar procesos de enseñanza aprendizaje al interior del centro educativo (Kaplan, 2004; Prieto, 2008). Generalmente, estos directivos conviven en una cultura escolar saturada de creencias asociadas al enfoque pedagógico por transmisión, que obviamente permea su pensamiento como líder. Esta

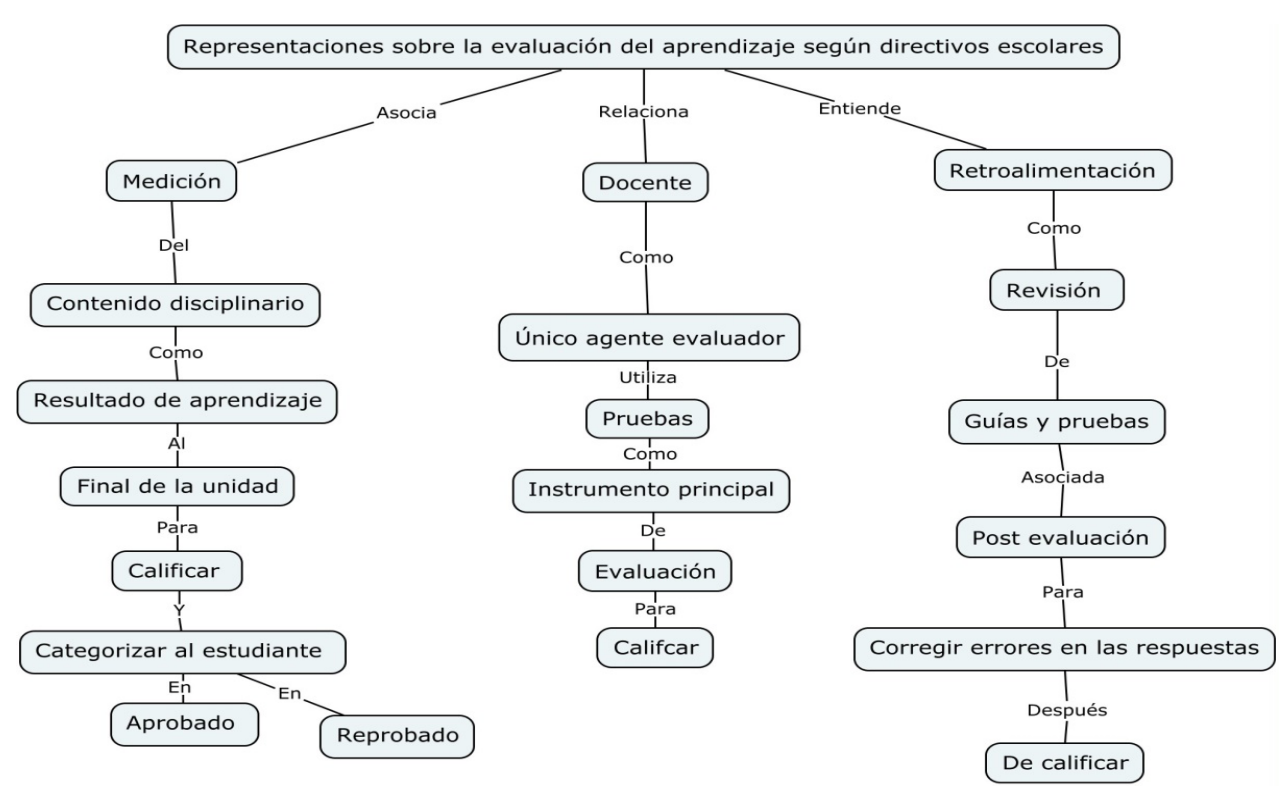

Figura 4. Representaciones sobre la evaluación del aprendizaje, según directivos escolares.

Fuente: Elaboración propia de los autores (2016). 
forma de pensar de los equipos directivos subyace a los lineamientos pedagógicos que orientan el accionar educativo y podrían influir en el profesorado en reproducir diseños de enseñanza descontextualizados, con escasas oportunidades de aprendizaje significativo para el estudiante.

En relación a las creencias que sostienen estos líderes escolares sobre el proceso de enseñanza aprendizaje, subyace una idea del aprendizaje escolar basada en la asimilación de contenidos fragmentados a través de un proceso repetitivo (Martínez y cols., 2009; García, 2010; Mellado \& Chaucono, 2015). Por otro lado, diversificar el proceso de enseñanza a las dificultades o particulares del estudiantado no parece un aspecto importante en sus argumentos (Joram, 2007). Estas afirmaciones, se corroboran en investigaciones que sostienen que las creencias son respuestas rutinarias del quehacer pedagógico, sin mayores cuestionamientos teóricos (Kaplan, 2004; Prieto, 2008). En otras palabras, estas representaciones directivas tradicionales tensionan la mejora continua de la organización, del desarrollo profesional docente, y por ende, del proceso de enseñanza aprendizaje en el aula.

Asimismo, recientes investigaciones sobre las creencias evaluativas de equipos profesionales del ámbito educativo han sostenido que todavía persisten algunas miradas punitivas de la evaluación, afirman que generalmente participa en el proceso solo el profesorado, y que utiliza la calificación al final de una unidad de enseñanza y/o periodo académico (Ahumada, 2005; Sanmartí, 2007; Pimineta, 2008; García, 2010). En este sentido, los planteamientos de los autores coinciden con los hallazgos de este estudio. En este caso, los directivos escolares argumentan desde una perspectiva tradicional, lejos de asumir la evaluación para o como aprendizaje. Por el contrario, se entiende como medición de resultados de parte del docente, también explican la retroalimentación como una instancia para corregir el error de contenidos que se evidencia recurrentemente en las evaluaciones sumativas.

En la búsqueda constante de avanzar en la línea investigativa de las creencias de directivos escolares, se indaga actualmente en develar las creencias que surgen desde el ejercicio del liderazgo pedagógico en las prácticas directivas al interior de los centros escolares.

A modo de conclusión, se puede sostener que a pesar de los avances a nivel mundial del liderazgo educativo, estos directivos escolares mantienen creencias arraigadas sobre diseño de enseñanza, proceso de aprendizaje y de evaluación que tienen implicancias en el liderazgo pedagógico que desarrollan los líderes en los centros educativos, particularmente en la gestión del desarrollo profesional docente y en el aseguramiento de la implementación del currículum.

Asimismo, se podría afirmar que las creencias develadas por este equipo directivo, pudiesen estar actuando como filtro del proceso de construcción de nuevos saberes y conocimiento pedagógico. Esta situación podría explicar el enfoque por transmisión de contenidos que declaran directivos escolares que participan de este estudio.

\section{Referencias}

Ahumada, P. (2005). Hacia una evaluación autentica del aprendizaje. México: Morata.

Amaya, R. (2007). La investigación en la práctica educativa: Guía metodológica de investigación para el diagnóstico y evaluación en los centro docentes. Madrid: Secretaría General Técnica.

Anderson, S. (2010). Liderazgo Directivo: Claves para una mejor escuela. Psicoperspectivas. Individuo y Sociedad, 9(2),34-54.

Barber, M. \& Mourshed, M. (2007). Cómo hicieron los sistemas educativos con mejor desempeño para alcanzar sus objetivos. Recuperado: 08 abr. 2016. Disponível: http://www.preal.org/

Bisquerra, R. (2004). Metodología de la investigación educativa. Madrid: La Muralla.

Bolívar, A. (2010). ¿Cómo el liderazgo pedagógico y distribuido mejora los logros académicos? Revisión de la investigación y propuesta. Magis, Revista Internacional de Investigación en Educación, 3(5), 79-106. Recuperado: 08 mar. 2016. Disponível: http://www.redalyc. org/articulo.oa?id=281023476005

Coll, C., Martin, E., Mauri, T., Miras, M., Onrubia, J., Solé, I., \& Zabala, A. (2007). El constructivismo en el aula. Madrid: Graó.

Contreras, S.A. (2010). Las creencias y actuaciones curriculares de los profesores de ciencias de secundaria de Chile (Tesis doctoral). Universidad Complutense de Madrid, Madrid, España). Recuperado: 08 mar. 2016. Disponível: http://eprints.ucm. es/11624/1/T32362.pdf

Díaz, C., Martínez, P., Roa, I., \& Sanhueza, M.G. (2010). Los docentes de la sociedad actual: Sus creencias y cogniciones pedagógicas respecto al proceso didáctico. Polis, Revista de la Universidad Bolivariana, 9(25), 421-436. Recuperado: 08 mar. 2016. Disponível: http://www.scielo.cl/pdf/polis/v9n25/art25.pdf

Elmore, R. (2010). Mejorando la escuela desde la sala de clases. Santiago de Chile: Fundación Chile.

García, M. R. (2010). Efecto de la formación inicial docente sobre la transformación de las creencias epistemológicas y de las creencias acerca de la buena enseñanza, de los estudiantes de pedagogíade la Pontificia Universidad Católica de Chile (Tesis de maestría). Pontificia Universidad Católica de Chile. Recuperado: 08 jan. 2016. Disponível: http://dspace2.conicyt.cl/bitstream/ handle/10533/76157/GARCIA_MARIA_1069M.pdf?sequence=1

Garbanzo, G. \& Orozco, V. (2010). Liderazgo para una gestión moderna de procesos educativos. Revista Educación. 32(1), 15-29.

Gómez, V. (2010). Explorando el cambio epistemológico y conceptual en la formación inicial de profesores en distintos contextos 
universitarios (Informe final Proyecto FONIDE). Chile: Ministerio de Educación, República de Chile.

Gómez, V., Muñoz, C., Silva, I., González, M. P., Guerra, P., \& Valenzuela, J. (2014). Creencias y oportunidades de aprendizaje en la práctica educativa en contextos de pobreza. Perfiles educativos, 36(144), 173-188. Recuperado: 03 mar. 2016. Disponível: http:// www.redalyc.org/pdf/132/13230751011.pdf

Joram, E. (2007). Clashing epistemologies: aspiring teachers' practicing teachers', and professors' beliefs about knowledge and research in education. Teaching and Teacher Education, 23(2), 123-135.

Jiménez, A.B. \& García, L.F. (2006). Pensar el pensamiento del profesorado. Revista Española de Pedagogía, 64(233), 105-122.

Kaplan, C. (2004). La inteligencia escolarizada. Buenos Aires: Amorrortu.

Krichesky, G. \& Murillo, J. (2011). Las comunidades profesionales de aprendizaje. Revista Iberoamerica sobre Calidad, Eficacia y Cambio en Educación, 9(1), 66-91. Recuperado: 08 mar. 2016. Disponível: http://www.rinace.net/reice/numeros/arts/vol9num1/art4.pdf

Leithwood, K., Harris, A. \& Hopkins, D. (2008). Seven strong claims about successful school leadership. School Leadership \& Management, 28(1), 27-42.

Macbeath, J. \& Nempster, N. (2009). Connecting Leadership and Learning. Principles for Practice. London: Routledge.

Martínez, R.D., Montero, Y.H. \& Pedrosa, M. (2009). Una aproximación a las creencias epistemológicas y pedagógicas de los profesores y a su valoración de internet. Revista Iberoamericana de Educación, 51(1), 1-15. Recuperado: 08 mar. 2016. Disponível: http://www. rieoei.org/deloslectores/3138MartInez.pdf.

Mellado, M. \& Chaucono, J. (2015). Creencias pedagógicas del profesorado de una escuela rural en el contexto mapuche. Actualidades Investigativas en Educación, 15(3), 1-19. Recuperado: 08 fev. 2016. Disponível: http://revistas.ucr.ac.cr/ index.php/aie/article/view/20924/21235
Mellado, M. \& Chaucono, J. (2016). Liderazgo pedagógico para reestructurar creencias docentes y mejorar prácticas de aula en contexto mapuche. Revista Electrónica Educare, 20 (1), 1-18. Recuperado: 08 mar. 2016. Disponível: http://www.revistas.una. ac.cr/index.php/EDUCARE/article/view/7526/7849

Ministerio de Educación, República de Chile. (2015). Marco para la Buena Dirección y el Liderazgo Escolar. Santiago de Chile: Centro de Perfeccionamiento, Experimentación e Investigaciones Pedagógicas.

Muñoz, G. \& Marfán, J. (2009). Formación y Entrenamiento de los Directores Escolares en Chile: Situación Actual, Desafíos y Propuestas de Política. Fundación Chile, 1-44.

Pimienta, J. (2008). Evaluación de los aprendizajes. Un enfoque basado en competencias. México: Pearson Educación.

Prieto, M. (2008). Creencias de los profesores sobre evaluación y efectos incidentales.

Caracas, Venezuela. Revista de Pedagogía 29(84), 123-144. Recuperado: 01 mar. 2016. Disponível: http://www.redalyc.org/articulo.oa?id=65908405

Rivera, L. (2010). Elementos conceptuales para el análisis de las prácticas de gestión educativa. En Guerra, Marcelino (coord.). Gestión de la Educación Básica. Referentes, reflexiones y experiencias de investigación. (1ª ed.) (pp. 71-86) México: SEPUPN.

Rojas, M.T. (2014). Las creencias docentes: Delimitación del concepto y propuesta para la investigación. Revista Electrónica Diálogos Educativos, 14(27), 89-112. Recuperado: 02 mar. 2016. Disponível: http://www.dialogoseducativos.cl/revistas/n27/rojas.

Sanmartí, N. (2007). 10 ideas claves: evaluar para aprender. Barcelona: Graó.

Wanlin, P. (2009). La pensée des enseignants lors de la planification de leur enseignement, Revue française de pédagogie, (166), 89128.

Recebido em: 08 de setembro de 2016 Aprovado em: 08 de fevereiro de 2017

\section{Sobre os autores}

María Elena Mellado Hernández (mmellado@uct.cl)

Doctora en Formación del Profesorado Facultad de Educación. Universidad Católica de Temuco, Chile.

Juan Carlos Chaucono Catrinao (juancarchau@yahoo.es)

Magíster en Gestión Escolar. Facultad de Educación. Universidad Católica de Temuco, Chile.

Carolina Pilar Villagra Bravo (cvillagra@uct.cl)

Magíster en Educación con mención Evaluación Educacional Facultad de Educación. Universidad Católica de Temuco, Chile. 